\title{
Pregnancy-Associated Severe Sepsis: Contemporary State and Future Challenges
}

Lavi Oud

To view enhanced content go to www.infectiousdiseases-open.com

Received: July 11, 2014 / Published online: September 9, 2014

(C) The Author(s) 2014. This article is published with open access at Springerlink.com

\section{ABSTRACT}

Pregnancy is associated with an increased risk of infection related to its associated mechanical and physiological changes. Sepsis remains among the top causes of maternal death worldwide and is associated with substantial maternal morbidity. However, there are sparse data on pregnancy-associated severe sepsis (PASS), related in part to infrequent reports, varying case definitions and methodological approach, small cohort size, and often limited focus on severe sepsis in selected phases of pregnancy outcomes. Available reports vary, but indicate that PASS is a rare but likely increasing complication, and it is more likely to develop with increased maternal age, among minority women, the poor, those lacking health insurance, those with chronic illness or

Electronic supplementary material The online version of this article (doi:10.1007/s40121-014-0037-7) contains supplementary material, which is available to authorized users.

L. Oud $(\bowtie)$

Division of Pulmonary and Critical Care Medicine, Department of Internal Medicine, Texas Tech

University Health Sciences Center at the Permian

Basin, Odessa, TX, USA

e-mail: lavi.oud@ttuhsc.edu pregnancy-associated complications, and following invasive procedures. Obstetric sites of infection are the most prevalent, but nonobstetric infections often underlie pregnancyassociated severe sepsis, though the source of infection is often not readily apparent during initial care. Women with PASS can have a rapidly fatal course and require heightened clinician vigilance for early diagnosis and timely effective intervention. Nevertheless, available reports raise concerns about prevalent substandard care of these patients, contributing to adverse outcomes. The case fatality of PASS appears lower than that in the general population with severe sepsis, while the long-term outcomes of survivors remain unknown.

Keywords: Intensive care unit; Mortality; Pregnancy; Resource utilization; Resuscitation; Severe sepsis

\section{INTRODUCTION}

The development of incremental morbidity and progression to death among infected patients 
has been a familiar part of physicians' practice long before the microbial etiology was discovered. However, the transformation in our understanding of a major part of the clinical spectrum of infection-related illness to include a systemic response to infecting microorganisms has been a relatively recent event, with the first attempt to standardize descriptive terminology and its definitions reported in 1992 by Bone et al. [1].

Sepsis is currently defined as a syndrome reflecting patient's systemic response to an infection [2]. A key component of this systemic response, though only part of the spectrum of sepsis manifestations has been the termed systemic inflammatory response syndrome (SIRS) [1, 2]. A septic patient is considered in turn to have severe sepsis if an infection-related organ dysfunction is present. Martin et al. [3] estimated that severe sepsis was present in about $34 \%$ of septic patients in the period of 1995-2000.

The incidence of severe sepsis is rapidly increasing and it is associated with high morbidity and mortality. It was estimated that in 2007 more than 780,000 adults (343 per $100,000)$ in the United States (US) developed severe sepsis [4] with an annual increase in rate of nearly $18 \%$ [5]. The global burden of sepsis has been estimated by Adhikari et al. [6] to range from 15 to 19 million cases per year.

The most common infection sites in severely septic patients are respiratory, genitourinary and abdominal [5, 7]. More than half of patients with severe sepsis have 2 or more organ failures (OFs) [4, 5], with pulmonary, renal, and circulatory systems most commonly affected [4]. It has been estimated that about half of the patients with severe sepsis in the US receive care in the intensive care unit (ICU) [7].

The annual death toll of severe sepsis in the US was estimated to exceed 210,000 patients per year in 2007, increasing nearly $180 \%$ since 2000 [4]. In addition, survivors of severe sepsis face long-term consequences of increased mortality rate and reduced quality of life [8]. The toll of severe sepsis varies with patients' demographics [9-11] and can be adversely affected by the type of health insurance [12]. The daily cost of care of septic patients is consistently higher than those without sepsis at all levels of care [13]. A recent report estimated that septicemia is the most expensive condition among hospitalized patients in the US [14].

Despite its increasing incidence and the personal and economic burdens, major strides were made over the past decade in improving the outlook for patients with severe sepsis. A landmark study by Rivers et al. [15] introduced the concept of early goal-directed therapy (EGDT), demonstrating marked mortality benefit of early recognition and targeted circulatory resuscitation in the Emergency Department. In addition, Kumar et al. [16] demonstrated that early administration of appropriate antibiotics is associated with decline in mortality of patients with septic shock, while mortality increased by $7.6 \%$ (absolute risk) with each hour of delay. These two reports were incorporated as part of a guideline by the surviving sepsis campaign (SSC), a multinational collaboration of multidisciplinary professional organizations, aiming to increase clinicians' and public awareness and reduce mortality due to severe sepsis [17]. Indeed, incorporating SSC guidelinebased bundled care into clinical practice was associated with reduced mortality [18].

The aforementioned strides have not been fully realized in the obstetric population. Pregnancy is associated with increased risk of infection, in part due to various pregnancyrelated mechanical and physiological changes [19]. In addition, recent evidence suggests that 
pregnancy is associated with an immunological shift away from inflammatory processes and inflammatory cytokines and toward a more anti-inflammatory immunologic state [20]. These changes may also play a role in the maternal response to overwhelming infection and subsequent sepsis [20]. In the 19th century, infection was the most common cause of maternal mortality, accounting for $50 \%$ of all maternal deaths [21]. While there has been tremendous progress in reducing maternal morbidity and mortality related to pregnancyassociated infectious complications, the latter remain a major source of pregnancy-related mortality in both developing and developed countries worldwide, reported to be the third to fourth most common cause of maternal death [22]. A recent review conducted by the World Health Organization has estimated the global burden of maternal sepsis to be more than $6,900,000$ cases per year [22].

Among the more basic ongoing challenges in our understanding the burden of pregnancyassociated sepsis and development of severe sepsis among infected patients, many investigators have noted that clinical reports often employ imprecise and variable terminology (often interchangeably) in use of terms such as septicemia, sepsis, septic shock, puerperal infection, puerperal fever, or maternal sepsis [23-26], thus affecting both clinical practice and present knowledge about maternal sepsis and severe sepsis in the obstetric population. Despite the voluminous body of published research on pregnancyassociated infections and sepsis, our contemporary understanding about pregnancy-associated severe sepsis (PASS) remains sparse.

There are several explanations for this knowledge gap. These include the following limitations of available data: (1) Published reports to date rarely focused explicitly and/or primarily on PASS. (2) When reported, studies often varied in their case definition of severe sepsis, at times at variance with those used in the general population, limiting inference and comparison across studies or with the general population. (3) Varying methodological approaches were used in studies of pregnancyassociated sepsis, further limiting comparisons across studies. (4) Sample size of reported PASS patients has been commonly small and often reflected local rather than population-level data, further limiting inferences from provided data. (5) Reports on PASS focused at times on selected periods of pregnancy (i.e., delivery), affecting inference about the burden of PASS across the full spectrum of pregnancy.

The focus of the following review is to examine the contemporary knowledge of the epidemiology, demographics, clinical characteristics, management approach, resource utilization, and outcomes of women affected by PASS, and to outline the present and future challenges in enhancing our insight and improving care and outcomes of this often devastating complication of pregnancy. The care of the fetus and fetal outcomes among patients with PASS is not part of the present review and has been described elsewhere [25].

\section{METHODS}

Relevant English-language original publications were sought through search of PubMed and EMBASE (from January 1992 through March 2014), using the following key terms: sepsis, severe sepsis, septic shock, septicemia, organ failure, critical illness, critical care, intensive care, mortality and pregnancy, abortion, delivery, puerperium, and miscarriage. Identified citations were further searched for additional referenced citations. The following 
publication categories were excluded: (a) published only in an abstract form, (b) contained no original data, or (c) did not specifically describe a group of patients with severe sepsis associated with pregnancy (i.e., at the minimum, the number of affected patients, with or without other characteristics), either as primary or additional focus of the report. The search strategy is described in detail in the Electronic Supplementary Material. Following removal of duplicate citations, 4,718 articles were identified, of which 4,710 did not meet eligibility criteria [reviews (322), reports on fetal/newborn events $(1,933)$, case reports (743), and lack of specific description of maternal severe sepsis $(1,712)]$. The remaining eight full-text articles were the focus of the present review. Descriptive statistics were used. This article does not involve any new studies with human or animal subjects performed by the author.

\section{THE EPIDEMIOLOGY OF PREGNANCY-ASSOCIATED SEVERE SEPSIS}

The key characteristics of identified studies providing epidemiological data on PASS are presented in Table 1. Several single-center and regional studies have reported the incidence of PASS. Mabie et al. [27] reported the incidence of pregnancy-associated septic shock of 12 per 100,000 deliveries-years in a two-hospital study. In a regional study, including 25 hospitals in the United Kingdom (UK) reported by Waterstone et al. [28], the incidence of PASS was 35 per 100,000 deliveries-years. Finally, a study of PASS in a tertiary center in Scotland by Acosta et al. [29] found an incidence of PASS 13 per 100,000 maternities-years. All three studies employed contemporary definitions of severe sepsis. Their findings have, however, several limitations. Data from local facilities may not reflect the epidemiology in a broader population. In addition, the sample size was extremely small, being 18 patients [27], 17 patients [28], and 14 patients [29], affecting precision of overall and annual [29] incidence estimates. Moreover, the reported incidence data were spread over 11 years [27] and 23 years [29], during which the development of PASS and obstetric practice have likely changed. In addition, the last two studies [28, 29] may have underestimated the number of PASS events, due to a restriction of case definition to culture-positive patients. However, studies of severe sepsis in the general population found that about 1 in 4 patients with severe sepsis can be culture-negative [15]. Finally, the incidence figures of these three studies are overstated in part due to use of delivery and maternity denominators in patients with PASS in the context of all pregnancy outcomes (i.e., abortion), rather than the total number of pregnancies among women at risk during study period.

Three population-level studies on PASS have been recently reported. Kramer et al. [30] have performed a retrospective analysis of a prospective national cohort in the Netherlands on severe maternal morbidity. The incidence of PASS was 21 per 100,000 deliveries-years. However, the validity of this estimate is limited by numerous methodological problems. There has been no explicit definition of sepsis, and severe sepsis was defined in part by admission to an ICU or any case of (an undefined) sepsis a physician considered to be severe morbidity. Specific OF/ dysfunction criteria were not used, which may have led to misclassification and overestimation of PASS incidence, as not all ICU admissions with an infection are due to severe sepsis. Indeed, as noted in a report by Afessa et al. 
Table 1 Key characteristics of studies providing epidemiological data on pregnancy-associated severe sepsis

\begin{tabular}{lllll}
\hline References & $\begin{array}{l}\text { Years of } \\
\text { study }\end{array}$ & Type/Country & $\begin{array}{l}\text { Number of } \\
\text { patients }\end{array}$ & $\begin{array}{l}\text { Scope of pregnancy } \\
\text { outcomes }\end{array}$ \\
\hline Mabie et al. [27] & $1986-1997$ & Local/US & 18 & All \\
Waterstone et al. [28] & $1997-1998$ & Regional/UK & 17 & $\begin{array}{l}\text { All deliveries after 24 weeks } \\
\text { of gestation }\end{array}$ \\
Acosta et al. [29] & $1986-2008$ & Local/UK & 14 & All \\
Kramer et al. [30] & $2004-2006$ & National/Netherlands & 78 & All \\
Acosta et al. [32] & $2005-2007$ & State/US & $791^{\mathrm{a}}$ & Live birth hospitalizations \\
Bauer et al. [33] & $1998-2008$ & National/US & $4,158^{\mathrm{a}}$ & Delivery hospitalizations \\
\hline
\end{tabular}

UK United Kingdom, US United States

${ }^{a}$ Number of hospitalizations

[31], studying obstetric patients in the ICU, among all obstetric sepsis patients admitted to the ICU, $49 \%$ did not have severe sepsis, when the authors used the consensus definitions [1]. In addition, as acknowledged by the investigators, sepsis was not a pre-defined condition for the prospective data collection, leading to possible underestimation of PASS events [30]. The number of PASS patients was only 78, limiting further the precision of incidence estimates. Finally, although PASS events spread over all pregnancy outcomes, the denominator used for incidence estimates was the number of deliveries which, as noted above, may have overestimated the actual incidence.

A more recent study by Acosta et al. [32] examined administrative data of live birth hospitalizations in the state of California. The reported incidence of PASS was 49 hospitalizations per 100,000 live births-years. The investigators included hospital length of stay $\geq 90$ th percentile and/or admission to ICU as part of case definition of severe sepsis, while not including OF criteria. It is thus likely that a substantial number of hospitalizations in that cohort did not have PASS, resulting in overestimation of PASS incidence. As noted earlier, admission to the ICU is not a reliable surrogate for diagnosis of PASS [31]. A misclassification of patients in this cohort is further supported by the markedly low rate of separately reported, selective (undefined) OFs (respiratory failure in 10.5\%), hospital length of stay markedly lower than reported by others [27, 30], and an implausibly low hospital morality rate $(0.8 \%$ among non-shock patients) [32].

Finally, in the largest cohort reported to date, Bauer et al. [33] have examined a national administrative data set in the US, focusing on PASS among delivery hospitalizations. The incidence of PASS was 9 hospitalizations per 100,000 deliveries-years [33].

The broad range of reported estimates of PASS incidence in the aforementioned studies limits our understanding of the contemporary burden of severe sepsis on the obstetric population, even when considering only population-level investigations, and expected country-specific variation. As noted, varying case definitions and related methodological problems affect interpretation of the reported findings. In addition, the optimal code-based [i.e., International Classification of Diseases, Ninth Revision, Clinical Modification (ICD-9- 
$\mathrm{CM}$ )] case definition of severe sepsis (commonly using both specific ICD-9-CM codes for severe sepsis and septic shock and a combination of sepsis/infection codes, combined with codes of OF) when using administrative data remains unsettled, with reported incidence estimates of severe sepsis in the general population varying by as much as 3.5 -fold across different coding approaches [34]. It is thus possible that the study reported by Bauer et al. [33], while using similar, more conservative, case identification approach to that in studies of severe sepsis in the general population, may have substantially underestimated the burden of PASS. Nevertheless, when different administrative case definitions of severe sepsis were used in the general population, all trended similarly over time [34]. The study by Bauer et al. [33] likely represents at present time the broadest report to date on PASS, with their findings suggesting that the incidence of PASS among women during delivery hospitalizations is markedly lower than that in the general population with severe sepsis [4].

The available contemporary reports on PASS have been restricted to Western Europe and the US. However, as noted earlier [22], the bulk of the global burden of maternal sepsis and thus of PASS is affecting disproportionately developing countries. Thus, data from developing countries (and other regions) are urgently needed to better understand the current epidemiology and the public health impact of PASS in these areas. Unfortunately, these types of investigations can be challenging, especially in resource-limited areas, which often lack sufficient local epidemiological expertise and consistent ability by the relatively limited number of clinicians to accurately diagnose and report these complications. In addition, other than the report by Kramer et al. [30], with its noted limitations, no population-level data reported on the epidemiology of PASS across the full spectrum of pregnancy outcomes, including induced abortion, miscarriage, antepartum and postpartum hospitalizations.

Only one study to date has described trends of the incidence of PASS. Bauer et al. [33] reported that the incidence of PASS rose 10\% per year between 1998 and 2008. The incidence of PASS increased from 7 to 14 hospitalizations per 100,000 deliveries over study period. However, the sources of rising incidence of PASS remain unclear. Several investigators have noted the rising incidence of conditions and procedures leading to maternal severe sepsis and septic shock, including rising maternal age, obesity, chronic illness, use of cesarean section, and use of invasive procedures [25]. While the aforementioned factors are well associated with risk of infection, their role in progression from infection to severe sepsis among obstetric patients has not been systematically examined. Indeed, the changes in the frequency of the aforementioned risk factors over time among the patients reported by Bauer et al. [33] have not been reported and require further study.

Only a few studies on the relative development of PASS across different phases of pregnancy have been reported and varied markedly across cohorts. PASS related to abortion was reported in 6\% [27] to 7\% [35]. Development of PASS during the antepartum period occurred between 33\% [30] and 73\% [35], while postpartum PASS events were noted to account for $20 \%$ [35] and up to $92.9 \%$ [29] of all PASS events. The marked differences in the relative occurrence of PASS across different phases and outcomes of pregnancy reported in the aforementioned studies likely reflects unique local population characteristics, selection bias, and the small sample size. Further larger population-level studies are 
needed to better understand the risk of PASS across non-delivery phases of pregnancy.

The demographic characteristics of women developing PASS varied with the studied populations. The average age reported ranged from 25.8 years [27] to 32 years [30]. The rate of PASS event in teens and among women older than 34 years was described infrequently, reported in $13.6 \%$ and $19.9 \%$, respectively [33]. Black women constituted between 7.1\% [29] and 56\% [27] of PASS cohorts in local studies and between about 9\% [32] and 21.2\% [33] in population-level reports, while Hispanic women were reported in 13\% [35] and 56.4\% [32] of PASS events, reflecting regional variations. Health insurance among US patients with PASS has been reported in two studies. Medicaid was the predominant health insurance $(49.8 \%)$ of women nationally in the study by Bauer et al. [33], with 3.6\% lacking health insurance. Acosta et al. [32] reported the combination of public health insurance/no insurance in $58.2 \%$ of PASS hospitalizations.

Only limited description of chronic comorbidities in PASS patients has been reported to date. Local reports [27-29], as well as a national study [30] did not provide clinical details on chronic illness. The population-based study by Acosta et al. [32] documented only occurrence of diabetes and chronic hypertension among live birth PASS hospitalizations. Bauer et al. [33] reported a broader but still selective range of chronic comorbidities, with the most common being congestive heart failure (6\%), systemic lupus $(1.5 \%)$, and chronic liver disease $(0.7 \%)$. However, the investigators provided no data on the overall frequency of any chronic comorbidity (of those examined) among PASS hospitalizations, limiting the inference on the overall burden of chronic illness from their findings.
Risk factors for the development of PASS were examined in several reports. Reported risk factors included maternal age $\geq 35$ years [30, 33], low income [30], black race [32, 33], Medicaid insurance [33] or public insurance/ no insurance [32], tobacco use [28] congestive heart failure [33], diabetes [32], hypertension [32], chronic liver disease [33], chronic kidney disease [33], systemic lupus [33], human immunodeficiency viral infection [33], preeclampsia [28, 32], induced labor [29, 30], cesarean section [28-30], premature rupture of membranes [30, 33], and retained products of conception [33]. Of note, obesity was not an independent risk factor for PASS in the study by Bauer et al. [33], possibly due to its underreporting $(1.8 \%)$ in their population. The aforementioned predictors identify subsets of obstetric patients requiring extra vigilance for prevention, early recognition and intervention for PASS. However, as noted by others, the risk factors for maternal sepsis are not wellunderstood [36].

\section{CLINICAL MANIFESTATIONS OF PREGNANCY-ASSOCIATED SEVERE SEPSIS}

The most common sites of infection among patients with PASS in local studies were described variably as involving the genital (39\%) [27] and urinary (37\%) [35] tracts. Kramer et al. [30] reported in their national study that genital tract infections were the most common, noted in $56 \%$ of their patients. No data on sites of infection were reported on PASS hospitalizations in the study by Acosta et al. [32]. Finally, in the national population study by Bauer et al. [33], the genital tract was the most common reported site of infection (56.7\%) among PASS hospitalizations. Of note, 
pneumonia was reported in $29.7 \%$ of PASS hospitalizations [33].

Although SIRS has been considered part of the bedside definition of sepsis in the general population, it was not validated in obstetric patients pre- or post-delivery and multiple investigators have raised concerns about the appropriateness of its cutoff values, which are often observed among otherwise healthy pregnant women [25]. The clinical findings of PASS include those related to a specific site of infection. Nevertheless, the site of infection is often not readily apparent in these patients. Indeed, Mabie et al. [27] have reported that the source of infection was not apparent in $44 \%$ of their patients with septic shock.

In addition, patients with PASS can display findings related to specific organ dysfunction or failure. Relatively limited data are available on the type, frequency, and number of failing organs among women developing PASS. Respiratory failure was the most common OF among PASS patients, reported in $44 \%$ [27] to $70 \%$ [35] in local studies, and $34 \%$ in a population study by Bauer et al. [33]. Renal failure was reported between $16 \%$ [33] to $37 \%$ [35]. Acosta et al. [32] did not describe systematically the occurrence of failing organs in their population. Hematological dysfunction was especially common, ranging between 39\% [27] to 43\% [35] of patients in local studies, and in $19 \%$ of PASS hospitalizations in a populationbased investigation [33]. Neurological dysfunction appears less common, described in $8 \%$ [33] of hospitalizations to $11 \%$ [27] of patients, although Snyder et al. [35] reported "altered mental status" in 30\% of their patients, without providing further detail. Only one study has reported systematically the distribution of the number of failing organs in PASS. Snyder et al. [35] found a single OF in
$40 \%, 2$ OF in $27 \%$ and $\geq 3$ OF in $33 \%$ of their patients.

Severe sepsis in the obstetric population can become rapidly fatal. Kramer et al. [30] noted that the time from the first symptom of infection to "full-blown sepsis" was $<24 \mathrm{~h}$ in $39 \%$ of their patients and that among women who died due to severe sepsis, the time from the onset of infection to death was less than $24 \mathrm{~h}$ in $50 \%$ of patients. Similarly, Snyder et al. [35] reported a rapid deterioration among all PASS patients who died. It has been further noted by some investigators that a predominant focus on genital tract sepsis may mislead clinicians in their assessment of pregnancy-associated infections [36]. These findings underscore the need for prompt recognition and timely effective intervention in patients with PASS. Because early clinical findings may overlap those of pregnancy-related physiological changes [25], while the site of infection may not be readily apparent [27], heightened level of suspicion by clinicians is crucial for adequate care of affected patients.

\section{MICROBIOLOGY OF PREGNANCY- ASSOCIATED SEVERE SEPSIS}

Patient-level data on the pathogens associated with PASS are limited due to the rarity of this complication in the obstetric population. Most of the available data on the antimicrobial management of PASS have been adapted from that on the microbiology among infected obstetric patients who are not necessarily severely septic. It is presently unknown to what extent these data apply to PASS population. When reported, microbiology data varied across studies. Escherichia coli was the most common isolate in the study by Mabie et al. [27], while group A streptococci 
dominated (32\%) the isolated pathogens in the study by Kramer et al. [30]. Microbial isolates were also reported in $40.4 \%$ of PASS hospitalizations by Bauer et al. [33]. Gramnegative and Gram-positive bacteria were evenly reported $(49.5 \%$ and $46.1 \%$, respectively). E. coli was the most common isolate. The investigators did not describe rates of polymicrobial versus monomicrobial PASS events.

Infections in the obstetric population are often described as polymicrobial [25], likely reflecting the predominance of genital tract infection. No data are presently available on site-specific infecting microorganisms in obstetric patients with sepsis versus severe sepsis. Similarly, contemporary trends in antimicrobial resistance of infecting microorganisms among patients with maternal sepsis and specifically PASS have not been systematically examined and require further study.

\section{MANAGEMENT OF PREGNANCY- ASSOCIATED SEVERE SEPSIS}

Early recognition of possible severe sepsis, coupled with timely effective interventions are key elements in the management of PASS, similarly to those in the general population with severe sepsis. Because, as noted earlier, the initial clinical manifestations of PASS may overlap those of pregnancy-related physiological changes $[24,25]$, while the findings pointing to the source of infection may not be readily apparent, heightened level of suspicion by clinicians is essential to assure timely care.

The specific components of care of patients with PASS are commonly based on the periodically revised practice guidelines of the SSC [17], which include evolving research data on severe sepsis. However, the SSC diagnostic criteria and care elements were never validated in the obstetric population and pregnant women were commonly excluded from severe sepsis trials [15, 37]. Early antimicrobial therapy, prompt circulatory resuscitation in patients with hypotension or elevated lactate, and effective early source control of infection are the main elements of the initial care of PASS, with further organ-specific support in individual patients. Patients with PASS are commonly managed in an ICU.

Empiric broad-spectrum antimicrobial therapy should be initiated within the first 60 min of the clinical manifestations of PASS [17] (once the patient is in a healthcare setting), adjusted for the suspected site of infection (if apparent) and selected with knowledge of the local antimicrobial resistance patterns of potential pathogens. A recent report by Ferrer et al. [38] has confirmed the earlier findings by Kumar et al. [16], demonstrating in a large multinational dataset that each hour of delay in antimicrobial therapy is associated with adjusted linear rise in patient mortality for both severe sepsis and septic shock [38]. The absolute risk of death with antibiotic delay was lower than that reported by Kumar et al. [16], likely reflecting in part the markedly reduced case fatality in contemporary severely septic patients and increased adherence to other components of the early support of these patients. Because genital tract infections are a common source of PASS, and obstetric infections tend to be polymicrobial [25], initial broad-spectrum antimicrobial therapy should include coverage of Gram-positive, Gram-negative, and anaerobic bacteria [25]. Blood and site-specific cultures should be obtained prior to staring antibiotics, but should not impede their timely administration. 
Circulatory resuscitation should be promptly started in hypotensive patients and in those with occult hypoperfusion, manifested by elevated serum lactate. Nevertheless, nearly $50 \%$ of hemodynamically unstable patients are not fluid-responsive (that is, do not show increase of their cardiac output or stroke volume in response to acute fluid resuscitation) [39] and recent reports indicate that increased positive fluid balance is associated with increased risk of death in patients with septic shock [40]. The dynamic rise of blood volume during pregnancy and its subsequent change postpartum [24] add to the complexity of targeted volume resuscitation of women developing PASS and underscore the need to assure appropriate circulatory volume support, while minimizing harm. Further studies are urgently needed to better define optimal circulatory volume resuscitation approach in obstetric patients with shock and specifically those developing PASS. Isotonic crystalloids are used for circulatory resuscitation of severe sepsis, as colloids (albumin) were not shown to be more beneficial [41], and starches should be avoided due to increased risk of acute kidney injury and mortality [17]. Catecholamines should be added for persistent hypotension despite intravenous volume resuscitation. Norepinephrine is considered the vasopressor of choice in septic shock [17] in the general population, but its role versus other vasopressors has not been systematically examined in the obstetric population.

As noted earlier, a protocolized resuscitative approach, EGDT [15], including placement of a central venous catheter and targeting resuscitation to achieve specific end-points of central venous pressure and central venous oxygen saturation, has been recommended in patients with overt shock or lactate levels $\geq 4 \mathrm{mmol} / \mathrm{l}$ [17]. However, a recent multicenter study of patients with septic shock [37] found that non-protocolized care can result in similar patient outcomes as EGDT or protocolized care, as long as there is early recognition of severe sepsis, and patients receive prompt administration of appropriate antibiotics, and early intravenous fluid resuscitation, coupled with remainder of the non-resuscitative care elements recommended by the SSC [17]. Respiratory and other systemic support should be provided depending on occurrence and severity of other organ dysfunction or failure [17]. Surgical or other interventional source control of infection should be provided early in selected patients with PASS. Mabie et al. [27] have reported the need for surgical intervention in $44.4 \%$ of their septic shock patients.

Mechanical ventilation is often required for severely septic patients developing respiratory failure. A lung-protective strategy has been recommended in patients with acute respiratory distress syndrome [17]. This approach involves among other components use of lower tidal volume and allowing "permissive hypercarbia". However, while avoiding excessively high, non-physiological tidal volume would likely be beneficial in mechanically ventilated obstetric patients, pregnant women were excluded from studies on the acute respiratory distress syndrome. Hypercarbia is generally well tolerated by nonobstetric, mechanically ventilated patients with acute respiratory distress syndrome and has been demonstrated to possibly have systemic organ-protective effects [42]. However, the balance between avoiding hypercarbia in mechanically ventilated pregnant patients and the adverse pulmonary and systemic consequences associated with overly aggressive augmented ventilation have not been 
determined in this population and require further study.

Among women with PASS developing prior to delivery, prompt initiation of fetal monitoring and consideration of timing and type of delivery should be integral parts of care. However, delivery was not shown to improve maternal outcomes among septic women [43]. The details of fetal care in women with severe sepsis have been described elsewhere [25].

While data on the general elements of care of severe sepsis in the general population and in PASS patients have been readily accessible to clinicians (in developed countries), many challenges remain in the care of PASS. Multiple investigators have described prevalent substandard care in women with PASS. Kramer et al. [30] have found that among women who died due to severe sepsis, a substandard care analysis showed delayed in diagnosis and/or therapy in $38 \%$ of patients. In the report of the confidential enquiry on maternal deaths in the UK, Cantwell et al. [44] reported that "substandard care" occurred in 69\% of patients. The authors recommended "going back to the basics", including among other recommendations, mandatory, audited training of all clinical staff in the identification and initial management of pregnancy-associated sepsis.

Because of the rarity of PASS, with an estimate of up to around 2,000 events per year in the US (when using the highest populationbased incidence data to date [32]), most clinicians and hospitals are unlikely to encounter even a single patient with PASS in a given year. The rarity of PASS, coupled with its demonstrated risk of a rapidly fatal course, underscores the ongoing challenges in assuring timely recognition and care of these high-risk patients.

\section{RESOURCE UTILIZATION IN PREGNANCY-ASSOCIATED SEVERE SEPSIS}

Patients with PASS are often managed in an ICU [27, 30, 31, 35]. Kramer et al. [30] reported ICU utilization in $79 \%$ of their patients with severe sepsis. However, as noted earlier, it is possible that septic patients without OF were included in their analysis, and ICU admission among septic obstetric patients is an inaccurate surrogate measure for a case definition of PASS [31]. None of the other reports on PASS described ICU utilization among the examined cohorts.

Use of life support interventions was not systematically described in available reports on PASS. Mechanical ventilation was used in $7.6 \%$ of PASS hospitalizations reported by Acosta et al. [32], although the reported rate is likely an underestimate due to the noted overly broad case definition of PASS. On the other hand, mechanical ventilation was used in $52 \%$ of septic shock hospitalizations reported in the same study, based on an "explicit" code-based definition of septic shock (i.e., use of only a specific ICD-9-CM code for septic shock, rather than including in addition a combination of codes for sepsis/infection and OF) [32]. Bauer et al. [33] described use of mechanical ventilation for $\geq 96 \mathrm{~h}$ in about $25 \%$ of their patients. Hemodialysis use was reported in about 5\% [33] of PASS hospitalizations to 10\% [35] of PASS patients. Further studies are required on the use of life support and other interventions in patients developing PASS.

Hospital length of stay among PASS patients was reported infrequently, ranging from 10 to 19 days in the study by Kramer et al. [30]. Acosta et al. [32] reported a relatively short median length of stay of 5 days in their nonshock PASS hospitalizations, likely reflecting 
case misclassification. The average ICU length of stay among survivors of septic shock was 15.1 days in the study by Mabie et al. [27]. None of the reports to date have addressed the fiscal toll of PASS. Further studies are needed to better understand the contemporary resource utilization in PASS patients.

\section{OUTCOMES OF PREGNANCY- ASSOCIATED SEVERE SEPSIS}

The case fatality of PASS has varied in available reports. When reported, data were restricted to hospital mortality. Among patients with septic shock, reported case fatality has ranged from $28 \%$ [27] to 33\% [35]. Using an "explicit" ICD-9CM code to define septic shock, Acosta et al. [32] reported case fatality of $14.3 \%$. Case fatality of PASS ranged from 10\% [35] to $17.6 \%$ [28] in local studies. Kramer et al. [30] reported case fatality of $7.7 \%$ in a national study of severe sepsis. As noted earlier, their findings should be interpreted with caution due to multiple methodological limitations. Similarly, an overly broad and non-specific case definition of PASS likely explains the remarkably low hospital mortality of $0.8 \%$ (1.8\%, including septic shock) reported by Acosta et al. [32]. In the largest study to date on PASS by Bauer et al. [33], the authors did not report the case fatality of PASS hospitalizations. Rather, they described case fatality of $3.2 \%$ for all maternal sepsis (i.e., both non-severe sepsis and PASS). The authors described a rising mortality rate by $10 \%$ per year, between 1998 and 2008 for all sepsis hospitalizations.

The reported case fatality of PASS appears markedly lower than that observed in the general population with severe sepsis $[4,5]$. Several investigators have suggested that younger age and the generally healthy obstetric population may explain these observations $[25,40]$. However, there have been no reports to date on direct comparisons between PASS patients and contemporaneous, similarly managed, age-similar, non-pregnant women with or without chronic comorbidities. Thus, it is unclear whether the low case fatality of PASS is related to a different response to infection and therapy in obstetric patients than among their non-pregnant and otherwise healthy counterparts.

The increasing mortality rate of all maternal sepsis, reported by Bauer et al. [33], likely reflects the increasing incidence of PASS reported by the investigators over study period. The authors noted that the incidence of overall sepsis remained stable, while both the incidence of PASS and sepsis-related mortality rate rose at the same annual rate [33]. While specific data were not provided by the investigators, their findings suggest a possibility of stable case fatality over study period. Moreover, other available reports do not clearly indicate decreasing case fatality of PASS over time. If the aforementioned postulate is correct, the results stand in sharp contrast with reports on severe sepsis in the general population, which have consistently reported decreasing case fatality over the past decade, possibly reflecting in part improved care, in an increasingly aging and sicker population $[4,5]$. Indeed, because the code-based approach used by Bauer et al. [33] to identify hospitalizations with severe sepsis was similar to that employed by other investigators in study of severe sepsis in the general population $[4,5]$, the findings of the former cannot be readily dismissed as caused by case misclassification. If the case fatality of PASS has remained unchanged, the source of this trend would require further investigation. The factors proposed for increasing the incidence of PASS (i.e., rising rates of obesity, older maternal age, and 
possibly increasing associated burden of chronic illness) may have contributed to the postulated lack of decrease in case fatality, though their rates among PASS hospitalizations were not trended over the study period examined by Bauer et al. [33]. However, the contemporary prevalent substandard care noted by other investigators [30, 35, 40], with delayed recognition and therapy in PASS patients, in contrast with the improving care practices in the general population with severe sepsis [18], has likely played a substantial part. None of the studies to date have described predictors of mortality of patients developing PASS, likely in part due the very small number of mortality outcomes inmost reports. Further research is required to better identify patients with PASS with increased risk of death to better target preventive and therapeutic interventions.

Severe sepsis can be associated with multiple long-term sequelae among survivors, including higher long-term mortality than that of the general population, lingering cognitive and physical dysfunction, as well as mental health sequelae, including depression, anxiety, and post-traumatic stress disorder [8, 45, 46]. None of the reports to date on PASS have described systematically the hospital disposition among survivors or their long-term clinical course. Further studies are urgently needed to better understand the post-hospitalization outcomes of survivors of maternal severe sepsis, to better address prevention and need for long-term care interventions.

\section{CONCLUSION}

PASS is a rare, but likely rising complication in some developed countries, while there is lack of data on its occurrence in developing countries. PASS has been infrequently described and multiple methodological limitations affect the interpretation of the varying epidemiological, clinical, resource utilization and outcome characteristics described by investigators to date. PASS is more likely to develop among minority women, the uninsured, those with chronic illness, and following invasive interventions. The genital tract is the most common reported site of infection. However, other, non-obstetric, sites of infection should be considered, though the site of infection may often not be readily apparent. Although the reported case fatality is lower compared with the general population with severe sepsis, PASS can be rapidly fatal. Because of the overlap between some of the early clinical manifestations of PASS and those of normal pregnancy-related physiological changes, and the rarity of this condition, high level of clinicians' vigilance is crucial for assuring early recognition and timely intervention. Future studies are urgently needed to better understand the burden of PASS across the spectrum of pregnancy outcomes, in both developed and developing countries, to improve systemic approach to assure effective care, and for improved insight into its longterm sequelae.

\section{ACKNOWLEDGMENTS}

No funding or sponsorship was received for this study or publication of this article. The author meets the ICMJE criteria for authorship for this manuscript, takes responsibility for the integrity of the work as whole and has given final approval for the version published.

Conflict of interest. Lavi Oud declares no conflict of interest. 
Compliance with ethics guidelines. Because we review publicly reported data, this study is exempt from formal review by the Texas Tech Health Sciences Center Institutional Review Board. This article does not involve any new studies with human or animal subjects performed by the author.

Open Access. This article is distributed under the terms of the Creative Commons Attribution Noncommercial License which permits any noncommercial use, distribution, and reproduction in any medium, provided the original author(s) and the source are credited.

\section{REFERENCES}

1. Bone RC, Balk RA, Cerra FB, et al. Definitions of sepsis and organ failure and guidelines for the use of innovative therapies in sepsis. The ACCP/SCCM Consensus Conference Committee. American College of Chest Physicians/Society of Critical Care Medicine. Chest. 1992;101:1644-55.

2. Levy MM, Fink MP, Marshall JC, et al. 2001 SCCM/ ESICM/ACCP/ATS/SIS International Sepsis Definitions Conference. Crit Care Med. 2003;31:1250-6.

3. Martin GS, Mannino DM, Eaton S, Moss M. The epidemiology of sepsis in the United States from 1979 through 2000. N Engl J Med. 2003;348:1546-54.

4. Kumar G, Kumar N, Taneja A, et al. Nationwide trends of severe sepsis in the 21st century (2000-2007). Chest. 2011;140:1223-31.

5. Lagu $\mathrm{T}$, Rothberg $\mathrm{MB}$, Shieh $\mathrm{M}$, et al. Hospitalizations, costs and outcomes of severe sepsis in the United States 2003-2007. Crit Care Med. 2012;40:754-61.

6. Adhikari NK, Fowler RA, Bhagwanjee S, et al. Critical care and the global burden of critical illness in adults. Lancet. 2010;138:1339-46.

7. Angus DC, Linde-Zwirble WT, Lidicker J, et al. Epidemiology of severe sepsis in the United States: analysis of incidence, outcomes, and associated costs of care. Crit Care Med. 2001;29:1303-10.
8. Winters BD, Eberlein M, Leung J, et al. Long-term mortality and quality of life in sepsis: a systematic review. Crit Care Med. 2010;38:1276-83.

9. Barnato AE, Alexander SL, Linde-Zwirble WT, Angus DC. Racial variation in the incidence, care, and outcomes of severe sepsis. Am J Respir Crit Care Med. 2008;177:279-84.

10. Melamed A, Sorvillo FJ. The burden of sepsisassociated mortality in the United States from 1999 to 2005: an analysis of multiple-cause-ofdeath data. Crit Care. 2009;13:R28.

11. Dombrovskiy VY, Martin AA, Sunderram J, Paz HL. Rapid increase in hospitalization and mortality rates for severe sepsis in the United States: a trend analysis from 1993 to 2003. Crit Care Med. 2007;35:1244-50.

12. O'Brien JM, Lu B, Ali NA, et al. Insurance type and sepsis-associated hospitalizations and sepsisassociated mortality among US adults: a retrospective cohort study. Crit Care. 2011;15:R130.

13. Moerer O, Plock E, Mgbor U, et al. A German national prevalence study on the cost of intensive care: an evaluation from 51 intensive care units. Crit Care. 2007;11:R69.

14. Torio CM, Andrews RM. National inpatient hospital costs: the most expensive condition by Payer, 2011. HCUP Statistical Brief \#160. August 2013. Agency for Healthcare Research and Quality, Rockville. Available from: http://www.hcup-us.ahrq.gov/ reports/statbriefs/sb160.jsp. Accessed May 7, 2014.

15. Rivers E, Nguyen B, Havstad S, et al. Early goaldirected therapy in the treatment of severe sepsis and septic shock. N Engl J Med. 2001;346:1368-77.

16. Kumar A, Roberts D, Wood KE, et al. Duration of hypotension before initiation of effective antimicrobial therapy is the critical determinant of survival in human septic shock. Crit Care Med. 2006;34:589-96.

17. Dellinger RP, Levy MM, Rhodes A, et al. Surviving sepsis campaign: international guidelines for management of severe sepsis and septic shock: 2012. Crit Care Med. 2013;41:580-637.

18. Levy MM, Dellinger RP, Townsend SR, et al. The surviving sepsis campaign: results of an international guideline-based performance improvement program targeting severe sepsis. Crit Care Med. 2010;38:367-74.

19. Fernández-Pèrez ER, Salman S, Pendem S, Farmer C. Sepsis during pregnancy. Crit Care Med. 2005; 33(suppl):S286-93. 
20. Robinson DP, Klein SL. Pregnancy and pregnancyassociated hormones alter immune responses and disease pathogenesis. Horm Behav. 2012;62: 263-71.

21. Loudon I. Death in childbirth: an international study of maternal care and maternal mortality 1800-1950. Oxford: Clarendon Press; 1993.

22. Dolea C, Stein C. Global burden of maternal sepsis in the year 2000. Evidence and Information for Policy, World Health Organization, Geneva, July 2003. Available from: http://www.who.int/ healthinfo/statistics/bod_maternalsepsis.pdf. Accessed May 31, 2014.

23. Bamfo JE. Managing the risks of sepsis in pregnancy. Best Pract Res Clin Obstet Gynecol. 2013;27:583-95.

24. Guinn DA, Abel DE, Tomlinson MW. Early goal directed therapy for sepsis during pregnancy. Obstet Gynecol Clin N Am. 2007;34:459-79.

25. Barton JR, Sibai BM. Severe sepsis and septic shock in pregnancy. Obstet Gynecol. 2012;120:689-706.

26. Dillen JV, Zwart J, Schuttle J, Roosmalen JV. Maternal sepsis: epidemiology, etiology and outcomes. Curr Opin Infect Dis. 2010;23:249-54.

27. Mabie WC, Barton JR, Sibai B. Septic shock in pregnancy. Obstet Gynecol. 1997;90:553-61.

28. Waterstone W, Bewley S, Wolfe C. Incidence and predictors of severe obstetric morbidity: casecontrol study. BMJ. 2001;322:1089-94.

29. Acosta CD, Bhattacharya S, Tuffnell D, et al. Maternal sepsis: a Scottish population-based casecontrol study. BJOG. 2012;199:474-83.

30. Kramer HMC, Schuttle JM, Zwart JJ, et al. Maternal mortality and severe morbidity from sepsis in the Netherlands. Acta Obstet Gynecol Scand. 2009; 88:647-53.

31. Afessa B, Green B, Delke I, Koch K. Systemic inflammatory response syndrome, organ failure, and outcome in critically ill obstetric patients treated in an ICU. Chest. 2001;120:1271-7.

32. Acosta CD, Knight M, Lee HC, Kurinczuk JJ, Gould JB, Lyndon A. The continuum of maternal sepsis severity: incidence and risk factors in a populationbased cohort study. PLoS One. 2013;8:e67175.

33. Bauer ME, Bateman BT, Bauer ST, Shanks AM, Mhyre JM. Maternal sepsis mortality and morbidity during hospitalization for delivery: temporal trends and independent predictors for severe sepsis. Anesth Analg. 2013;117:944-50.
34. Gaieski DF, Edwards JM, Kallan MJ, Carr BG. Benchmarking the incidence and mortality of severe sepsis in the United States. Crit Care Med. 2013;41:1167-74.

35. Snyder CC, Barton JR, Habli M, Sibai BM. Severe sepsis and septic shock in pregnancy: indications for delivery and maternal and perinatal outcomes. J Matern Fetal Neonatal Med. 2013;26:503-6.

36. Sriskandan S. Severe peripartum sepsis. J R Coll Physicians Edinb. 2011;41:339-46.

37. The ProCESS Investigators. A randomized trial of protocol-based care for early septic shock. N Engl J Med. 2014;370:1683-93.

38. Ferrer R, Martin-Loeches I, Phillips G, et al. Empiric antibiotic treatment reduces mortality in severe sepsis and septic shock form the first hour: results from a guideline-based performance improvement program. Crit Care Med. 2014;42:1749-55.

39. Marik PE, Lemson J. Fluid responsiveness: an evolution of our understanding. Br J Anaesth. 2014;112:617-20.

40. Boyd JH, Forbes J, Nakada T, Walley KR, Russell JA. A positive fluid balance and elevated central venous pressure are associated with increased mortality. Crit Care Med. 2011;39:259-65.

41. Caironi P, Tognoni G, Masson S, et al. Albumin replacement in patients with sepsis of septic shock. N Engl J Med. 2014;370:1412-21.

42. Laffey JG, O'Croinin D, McLoughlin P, Kavanagh BP. Permissive hypercarbia-role in protective lung ventilatory strategies. Intensive Care Med. 2004; 30:347-56.

43. Paruk F. Infection in obstetric critical care. Best Prac Res Clin Obstet Gynecol. 2008;22:865-83.

44. Cantwell R, Clutton-Brock T, Cooper G, et al. Saving mothers' lives: reviewing maternal deaths to make motherhood safer: 2006-2008. The eight report of the confidential enquiries into maternal deaths in the United Kingdom. BJOG. 2011;118(suppl1):1-203.

45. Iwashyna TJ, Ely EW, Smith DM, Langa KM. Longterm cognitive impairment and functional disability among survivors of severe sepsis. JAMA. 2010;304:1787-94.

46. Cuthbertson BH, Elders A, Hall S, et al. Mortality and quality of life in the 5 years after severe sepsis. Crit Care. 2013;17:R70. 